\title{
COMPOSICIÓN, RIQUEZA, DIVERSIDAD Y ABUNDANCIA DE AVES EN CUATRO ÁREAS VERDES DE SANTO DOMINGO
}

\section{Composition, richness, diversity and abundance of birds in four green areas of Santo Domingo}

\author{
Hodali Almonte-Espinosa
}

Museo Nacional de Historia Natural "Prof. Eugenio de Jesús Marcano”. Calle César Nicolás Penson, Plaza de la Cultura Juan Pablo Duarte, Santo Domingo, República Dominicana. h.almonte@mnhn.gov.do.

\section{RESUMEN}

Se presenta un análisis sobre la riqueza, diversidad y abundancia de aves en cuatro áreas verdes de la ciudad de Santo Domingo: Plaza de la Cultura Juan Pablo Duarte, Parque Mirador Sur, Jardín Botánico Nacional Dr. Rafael M. Moscoso y Parque Mirador Norte. El trabajo de campo fue realizado desde febrero del 2013 hasta noviembre de 2017. Utilizando puntos de conteo y tranceptos en franjas se registró un total de 3473 individuos, correspondientes a 70 especies y 37 familias. Del total de especies registradas, 7 son endémicas, 37 residentes, 20 migratorias y 6 introducidas. El Parque Mirador Norte, presentó el más alto valor en riqueza de especies (54), mientras que la Plaza de la Cultura Juan Pablo Duarte y el Parque Mirador Sur registraron los valores más bajos, con 35 y 38 especies, respectivamente. La mayor abundancia de individuos se registró en el Jardín Botánico Nacional (1 373) y la menor en el Parque Mirador Norte (562). El mayor valor de diversidad corresponde al Parque Mirador Norte $\left(\mathrm{H}^{\prime}=3.3\right.$ y $\left.\mathrm{D}-1=0.94\right)$, mientras que la Plaza de la Cultura presentó el valor más bajo $\left(\mathrm{H}^{\prime}=2.3\right.$ y D-1=0.89). Las especies registradas fueron agrupadas en ocho gremios tróficos, siendo el de las omnívoras el más numeroso. De acuerdo con los criterios de la Unión Internacional para la Conservación de la Naturaleza (UICN), cuatro de las especies se encuentran amenazadas y otra casi amenazada, siendo estas áreas de importancia relevante para su conservación en la ciudad de Santo Domingo.

Palabras clave: áreas verdes, Santo Domingo, aves, riqueza de especies, diversidad, abundancia, gremios alimentarios.

\section{ABSTRACT}

An analysis of the richness, diversity and abundance of birds in four green areas of Santo Domingo city is presented. These green areas are Plaza de la Cultura Juan Pablo Duarte, Parque Mirador Sur, Jardín Botánico Nacional Dr. Rafael M. Moscoso and Parque Mirador Norte. Fieldwork was conducted from February 2013 to November 2017. Using Point count and strip transects, a total of 3473 individuals were registered, corresponding to 70 species and 37 families. Of the total number of registered species, seven are endemic, 37 resident, 20 migratory and 6 introduced. Parque Mirador Norte presented the highest value of species richness (54), while Plaza de la Cultura Juan Pablo Duarte and Parque Mirador Sur recorded the lowest values with 35 and 38 species respectively. The greatest abundance of individuals was recorded in Jardín Botánico Nacional (1 373) and the lowest in Parque Mirador Norte (562). The highest diversity value corresponds to Parque Mirador Norte $\left(\mathrm{H}^{\prime}=3.3\right.$ and $\left.\mathrm{D}-1=0.94\right)$, while Plaza de la Cultura presented the lowest value $\left(H^{\prime}=2.3\right.$ and $\left.\mathrm{D}-1=0.89\right)$. The registered species were grouped into eight trophic guilds, the omnivores being the most numerous. According to the criteria of the International Union for the Conservation of Nature (IUCN), 4 species are threatened and one is almost threatened, these areas being of significant importance for their conservation in the city of Santo Domingo.

Keywords: green areas, Santo Domingo, birds, species richness, diversity, abundant, threatened species, trophic guilds. 


\section{INTRODUCCIÓN}

Los procesos de urbanización son una de las principales causas de la pérdida de biodiversidad a nivel mundial y se espera que la continua expansión y crecimiento de las áreas urbanas provoque grandes pérdidas de áreas naturales, lo cual implicaría una reducción considerable de la riqueza y diversidad en estas zonas (Chance y Walsh, 2006).

Estudios realizados sobre la avifauna urbana en países de zonas templadas, han documentado que tanto la diversidad como la riqueza disminuyen conformen se extienden estas áreas, ya que su crecimiento trae consigo la fragmentación de los hábitats naturales. Igualmente, se ha encontrado que las comunidades de aves en las ciudades son dominadas por unas pocas especies, algunas de las cuales son introducidas (Lancaster y Rees 1979; Fernández-Juricic y Jokimäki, 2001).

En ese mismo orden, numerosos estudios sugieren que las áreas verdes ubicadas en ciudades son un refugio importante para la preservación de la diversidad biológica originaria de la zona (Fernández-Juricic y Jokimäki, 2001; Carbón-Ramirez et al., 2011). Además, las áreas verdes que presentan una alta composición de plantas nativas y cuerpos de aguas asociados a estas, favorecen el establecimiento de comunidades con una alta riqueza y diversidad de aves.

Los estudios sobre estas comunidades recientemente han despertado el interés de ornitólogos de diversos países (Carbón-Ramirez et al., 2011; Castillo Palacios et al., 2014; Cárdenas et al., 2003; Maragliano et al., 2009; Vázquez Plass y Wunderle Jr., 2013). Sin embargo, en República Dominicana los estudios sobre comunidades de aves en áreas urbanas son escasos, conociéndose solo la publicación de listas de especies en el libro Ruta Barrancolí (Latta y Wallace, 2012), en el cual se incluyen 58 para el Jardín Botánico Nacional Dr. Rafael Ma. Moscoso y 66 para el Parque Mirador Norte. Lorenzo et al. (2014) publicaron un informe sobre la fauna del Parque Mirador Sur, reportando 40 especies, mientras que para la Plaza de la Cultura no hay un listado publicado a esta fecha.

Este estudio analiza las comunidades de aves de cuatro áreas verdes urbanas, contribuyendo así al conocimiento en cuanto a la riqueza, diversidad, abundancia, gremios tróficos y estado de conservación del grupo en estos sitios.

\section{OBJETIVO}

-Caracterizar y comparar las comunidades de aves en cuatro áreas verdes de Santo Domingo.

\section{MATERIALES Y MÉTODOS}

\section{Áreas de estudio}

Este trabajo se realizó en cuatro áreas verdes de Santo Domingo: Plaza de la Cultura Juan Pablo Duarte (PLCU), Parque Mirador Sur (PMSU), Jardín Botánico Nacional Dr. Rafael María Moscoso (JBNA) y Parque Mirador Norte (PMNO).

Plaza de la Cultura Juan Pablo Duarte. Está ubicada en el centro de la ciudad Santo Domingo, es un complejo que incluye varias instituciones: Museo Nacional de Historia Natural "Prof. Eugenio de Jesús Marcano", Museo de Historia y Geografía, Museo de Arte Moderno y Museo del Hombre Dominicano, así como la Biblioteca Nacional y el Teatro Nacional. Esta plaza es considerada como un área verde de recreo. Posee una extensión aproximada de $1000 \mathrm{~m}^{2}$. Actualmente, su vegetación está compuesta por plantas ornamentales, matorrales abiertos y arboles dispersos. 
Parque Mirador Sur. Se localiza en la avenida Mirador Sur, a una elevación de 30 m s.n.m. y ocupa una superficie de aproximadamente $7 \mathrm{~km}^{2}$. En este parque se encuentran variados ambientes, como formaciones rocosas, cuevas, manantiales subterráneos, matorrales y arboles dispersos. Representa uno de los más importantes conjuntos urbanos paisajísticos de Santo Domingo. El parque queda dispuesto en dirección este-oeste, está caracterizado por sus amplias zonas verdes dedicadas a la recreación y esparcimiento de la ciudadanía (Miembros del Consejo Nacional de Asuntos Urbanos, 2007).

Jardín Botánico Nacional Rafael María Moscoso. Se encuentra localizado en el sector Altos de Galá, a una elevación 70-80 m s.n.m. y ocupa una superficie aproximada de $2 \mathrm{~km}^{2}$. El clima es tropical, húmedo, con una precipitación media anual de $1366 \mathrm{~mm}$, una humedad relativa de 83.7 y una temperatura promedio de $25.8^{\circ} \mathrm{C}$. El suelo es de origen calizo, poco profundo y de escasa fertilidad. El relieve es levemente plano y con numerosas ondulaciones; en la parte sur es atravesado por la gran Cañada, la que contribuye a la formación de variados microclimas (Mejía y García, 1994; Miembros del Consejo Nacional de Asuntos Urbanos, 2007).

El JBNA fue creado en 1976, con el objetivo de preservar y dar a conocer la flora nativa y exótica de la Hispaniola. En este se exhiben diferentes tipos de vegetación, desde plantas acuáticas, palustres, flotadoras y sumergibles, hasta palmáceas, orquídeas, bromelias, helechos y otras (Latta y Wallace, 2012). Además, en cumplimiento con los acuerdos internacionales, el $10 \%$ del área es una reserva forestal de la flora endémica y nativa (Miembros del Consejo Nacional de Asuntos Urbanos, 2007).

Parque Mirador Norte. Creado en 1996, queda ubicado en el municipio de Santo Domingo Norte, establecido por el Decreto No. 312-92. Constituye el área protegida más grande de la ciudad Santo Domingo, con alrededor de $10 \mathrm{~km}^{2}$. Es parte del cinturón verde de Santo Domingo. Es considerado como el principal pulmón de la ciudad y uno de los principales centros de recreo. Se caracteriza por poseer extensos bosques y galerías que cubren ambas márgenes del río Isabela en el tramo de su cruce por el parque; así como por lagunas, cañadas y humedales. Además, preserva una amplia porción de bosque húmedo, pero se desconoce el porcentaje exacto (Miembros del Consejo Nacional de Asuntos Urbanos, 2007).

\section{Métodos de muestreo, identificación y ordenamiento taxonómico}

El trabajo de campo se realizó desde febrero del 2013 hasta noviembre del 2017. Se utilizaron dos métodos de muestreo, seleccionados tomando en cuenta la cobertura forestal de las zonas de estudio. En el caso de la Plaza de la Cultura y el Parque Mirador Sur, que son áreas muy abiertas con algunos matorrales y arboles dispersos, fue usado el método de transectos en franjas, el cual consiste en realizar recorridos de 20 minutos, mientras son registradas todas las aves vistas y escuchadas (Ralph et al., 1995; Ralph et al., 1996). En la PLCU fueron establecidos cinco transectos, mientras que para el PMSU se establecieron diez, cada uno de 200 metros.

En el Jardín Botánico Nacional y el Parque Mirador Norte fueron establecidos 20 puntos de conteo, cada uno separado por una distancia mínima de aproximadamente $200 \mathrm{~m}$. Los datos fueron tomados en intervalos de diez minutos. Se identificaron todas las especies observadas y escuchadas (Ralph et al., 1995; Ralph et al., 1996).

El tiempo fijado para el levantamiento de los datos fue desde las 8:00 a.m. hasta las 11:00 a. m. Las observaciones se realizaron con binoculares Nikon 10 x 42 (10 de aumento y los objetivos de $42 \mathrm{~mm}$ de diámetro). Para la identificación de las especies se siguió a Latta et al. (2006) y para el ordenamiento taxonómico a The American Ornithologist Union (Banks et al. 2005, 2006, 2007, 2008; Chesser et al. 2009, 2010, 2014, 2017). 


\section{Tratamiento de los datos}

Con la finalidad de conocer mejor las comunidades en estudio, se usaron varios índices de diversidad y equidad de especies: índice de diversidad de Shannon $\left(\mathrm{H}^{\prime}\right)$, índice de Equidad de Pielou $\left(\mathrm{J}^{\prime}\right)$, índice de Dominancia de Simpson $(\lambda)$ y recíproco de Simpson (D-1), siempre de acuerdo a Moreno (2001). Con el objetivo de conocer los gremios alimentarios de las comunidades de aves en estudio, se procedió a agruparlas según su dieta, siguiendo a GonzálezSalazar et al. (2014).

\section{RESULTADOS Y DISCUSIÓN}

Riqueza y abundancia de especies. Se registró 3473 individuos y 70 especies de aves, distribuidas en 37 familias. Del total de especies registradas, siete son endémicas, 37 residentes, 20 migratorias, y cinco introducidas, una doméstica entre estas últimas (Tabla I).

La mayor abundancia se registró en el Jardín Botánico Nacional Dr. Rafael M. Moscoso (JBNA) con 1373 individuos, mientras que el Parque Mirador Norte (PMNO) presentó la menor, con 562 individuos (Tabla I). En los cuatro parques urbanos estudiados, las especies más abundantes y comunes fueron la Cigua Palmera (Dulus dominicus) con un 16\% de total registrado, la Paloma Común (Columba livia) con 10\%, el Pájaro Carpintero (Melanerpes striatus) con, 9.8\% y la Cigüita Común (Coereba flaveola) con $10.1 \%$. Estos datos concuerdan con resultados de otros autores (Lancaster y Rees, 1979; Fernandez-Juricic y Jokimäki, 2001), quienes sugieren que las comunidades de aves en áreas urbanas están dominadas por unas pocas especies, algunas de las cuales son introducidas.

Se destaca el registro del Degollado (Pheucticus ludovicianus), un ave migratoria poco común, reportada anteriormente en el Parque Nacional Los Haitises, Puerto Plata, Oviedo y en la Sierra de Bahoruco (Latta et al., 2006). También fue observado un ejemplar de Paloma Turca (Patagioenas squamosa), esta especie actualmente está restringida al bosque húmedo latifoliado, en áreas remotas, debido a esto se considera como un registro raro en Santo Domingo (Latta et al., 2006; Tabla I).

La riqueza de especies varió entre los parques, encontrándose que las áreas con mayor remanente de la vegetación nativa, como el PMNO y el JBNA, presentaron una alta riqueza de especies, con 54 y 47, respectivamente; en cambio, PLCU y PMSU, que son áreas abiertas con algunos matorrales y arboles dispersos, presentaron riqueza de especies menor, con 35 y 38, respectivamente (Tabla II). Estos valores solo incluyen a aves endémicas, residentes y migratorias, quedando excluidas las especies introducidas.

En el caso del JBNA y PMNO, los valores de riqueza registrados son similares, esto se podría explicar en relación a que en ambas áreas, además de conservar un importante remanente de vegetación nativa, hay cuerpos de agua: cañadas, lagos, fuentes permanentes y ríos. Además, el área muestreada fue de 200 hectáreas en cada parque. Al poseer una amplia variedad de ambientes, estas áreas favorecen la presencia de especies que no suelen encontrarse en áreas urbanas céntricas, tales como aves asociadas a cuerpos de agua y otras con requerimientos específicos.

De acuerdo con trabajos realizados en zonas templadas, los parques más grandes favorecen el establecimiento de un alto número de especies, debido a una mayor disponibilidad y diversidad de hábitats (Hinsley et al., 1995), esto contrasta parcialmente con lo encontrado en el PMSU, el que fue muestreado en su totalidad y presenta una menor riqueza de especies, pero posee una menor disponibilidad y diversidad de ambientes que el JBNA. 
Diversidad. La diversidad de especies en los parques urbanos evaluados varía, encontrándose que PMNO y JBNA mostraron los valores más altos del índice de Shannon: $\mathrm{H}^{\prime}=3.30$ y $\mathrm{H}^{\prime}=3.00$, respectivamente (Tabla II). El PMNO presentó el valor más bajo de dominancia de especie $(\lambda=0.05)$, siendo su recíproco (D-1) 0.94 , mientras que JBNA presentó el más alto $(\lambda=0.08) \mathrm{y}$ su recíproco fue 0.92. Estos últimos resultados se deben a la dominancia de especies como la Cigua Palmera (Dulus dominicus), la Paloma Común (Columba livia), el Pájaro Carpintero (Melanerpes striatus) y la Cigüita Común (Coereba flaveola).

En estudios realizados en áreas verdes urbanas de países templados, se ha encontrado que los lugares que poseen una alta diversidad y densidad de plantas nativas, tienden a presentar una alta diversidad biológica. Esto se aplica tanto PMNO como JBNA, ya que cada uno posee una amplia reserva forestal, siendo esta igual al 10\% de la superficie en el caso de JBNA, y probablemente sea mayor en PMNO, pero se desconoce con exactitud el porcentaje preservado en este último sitio.

Al comparar PMNO con los demás sitios se encontró una mayor variedad de ecosistemas, tanto acuáticos como terrestres, entre los cuales se incluyen remanentes del bosque húmedo, humedales, cañadas y lagunas, condición que podría favorecer una alta diversidad de aves (Talla II).

Los resultados obtenidos con el índice de equidad de Pielou sugieren que la comunidad de aves presente en PMNO posee una mayor homogeneidad (Tabla II), esto se refleja en los índices de diversidad utilizados, ya que usualmente a mayor equidad mayor diversidad de especies.

Gremios alimentarios. Las aves registradas fueron agrupadas en ocho gremios, siendo los omnívoros (21) los mejor representados, seguidos de los insectívoros (17), granívoros (9), carnívoros (3), nectarívoros (3), frugívoros (2) y carroñeros (1). En las cuatro áreas de estudio, el gremio dominante corresponde a las aves oportunistas que pueden consumir una amplia variedad de recursos, incluyendo restos de alimentos dejados por los visitantes. 


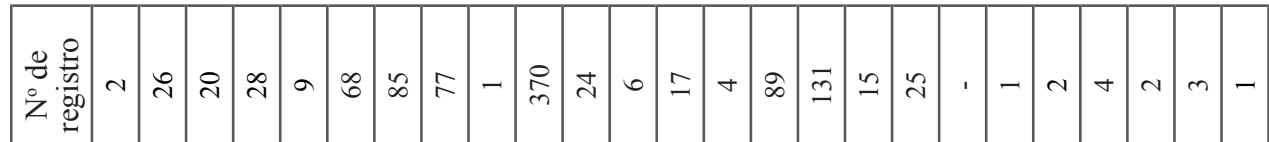

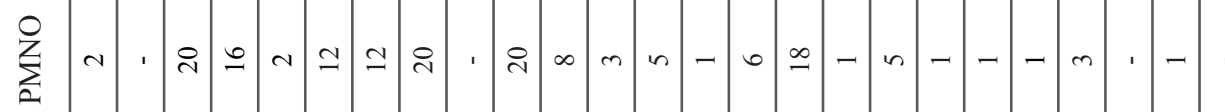

$\underset{\widetilde{z}}{\mathbb{z}^{\circ}, ~}$

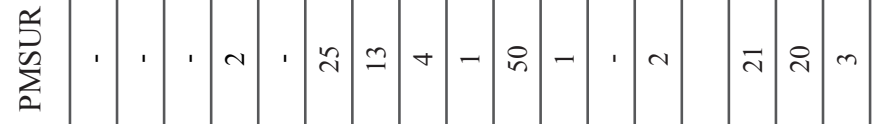

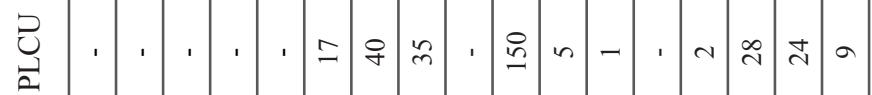

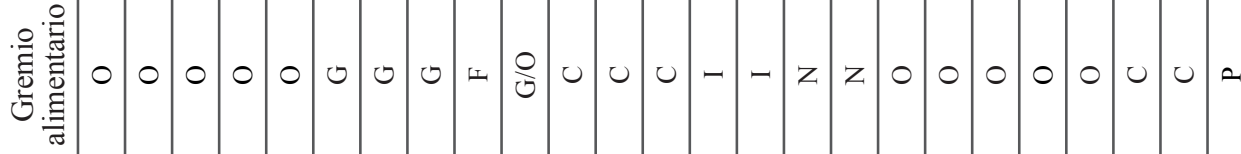

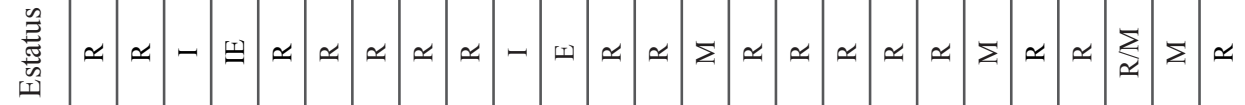

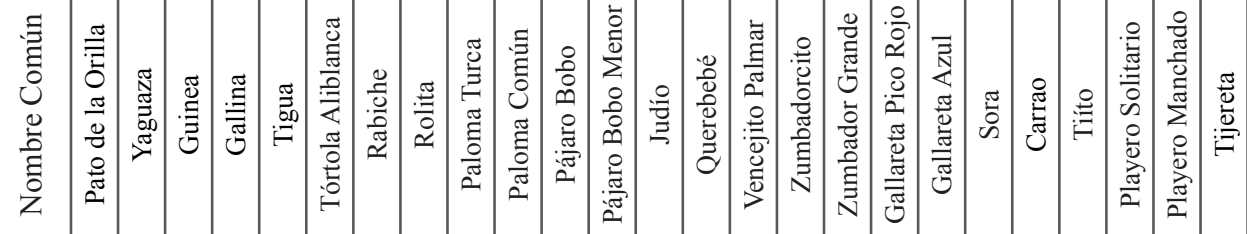

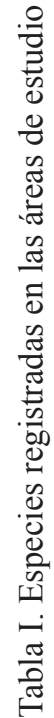

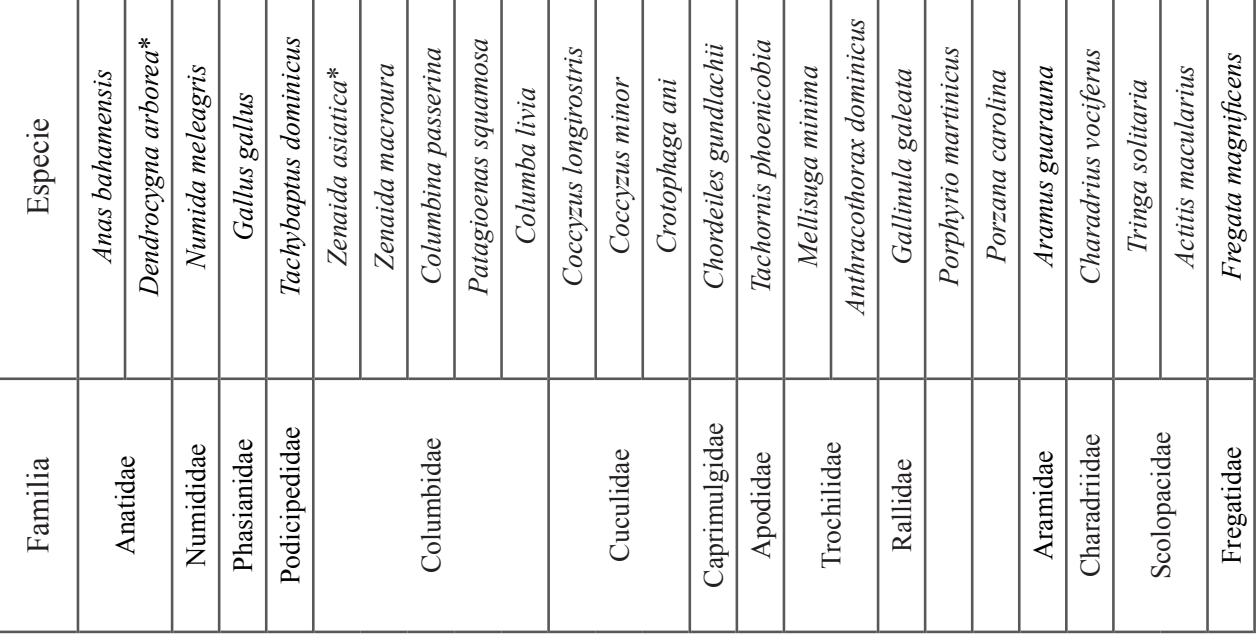




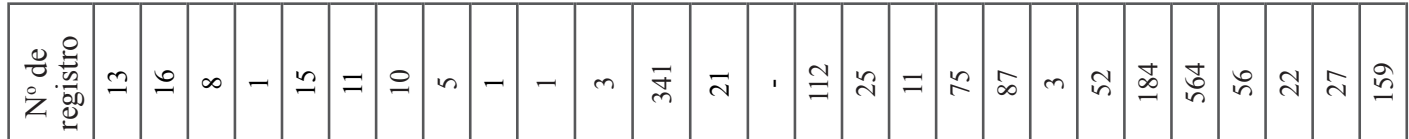

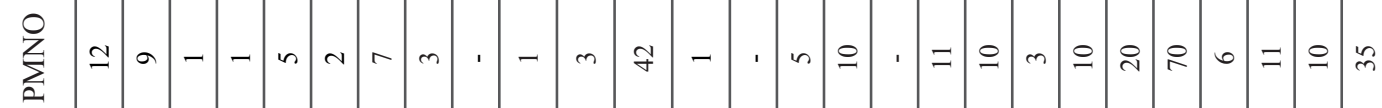

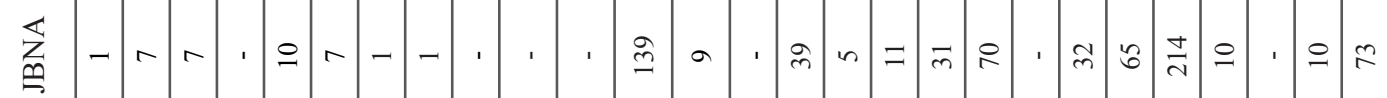

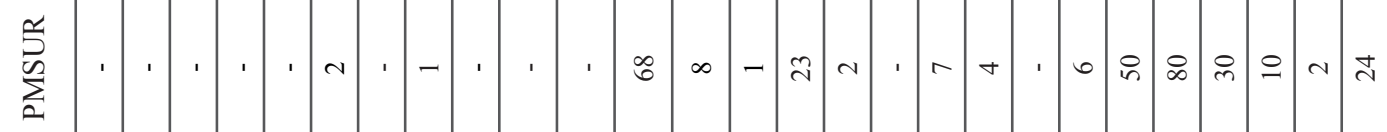

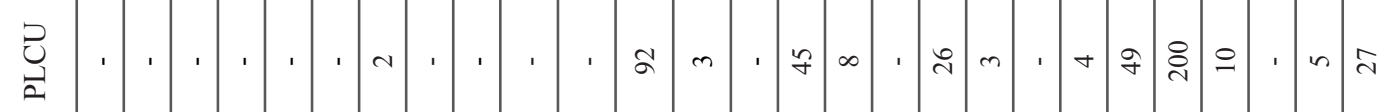

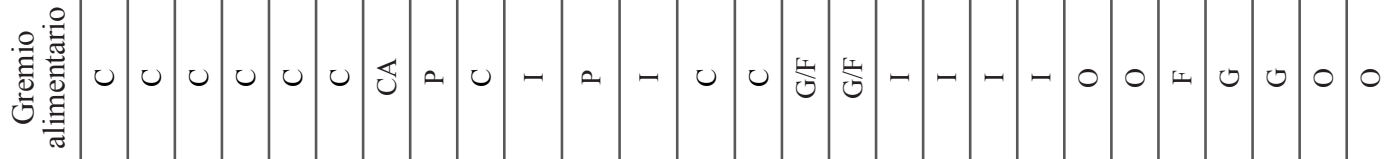

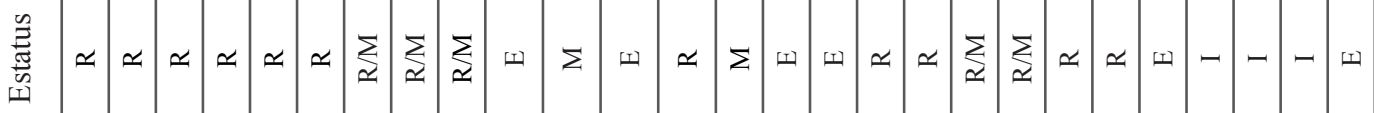

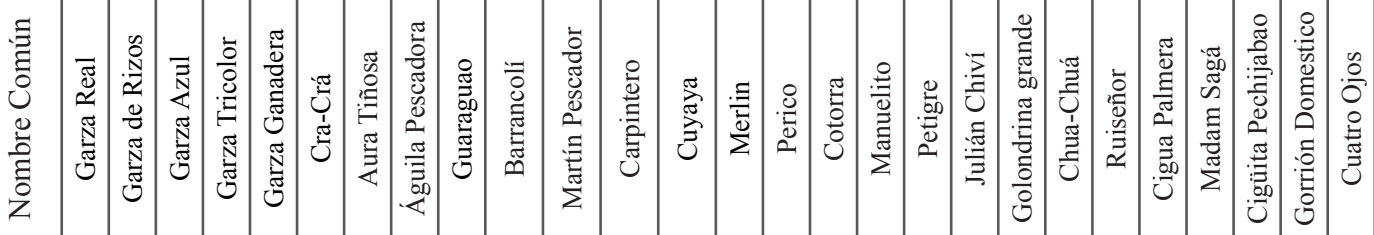

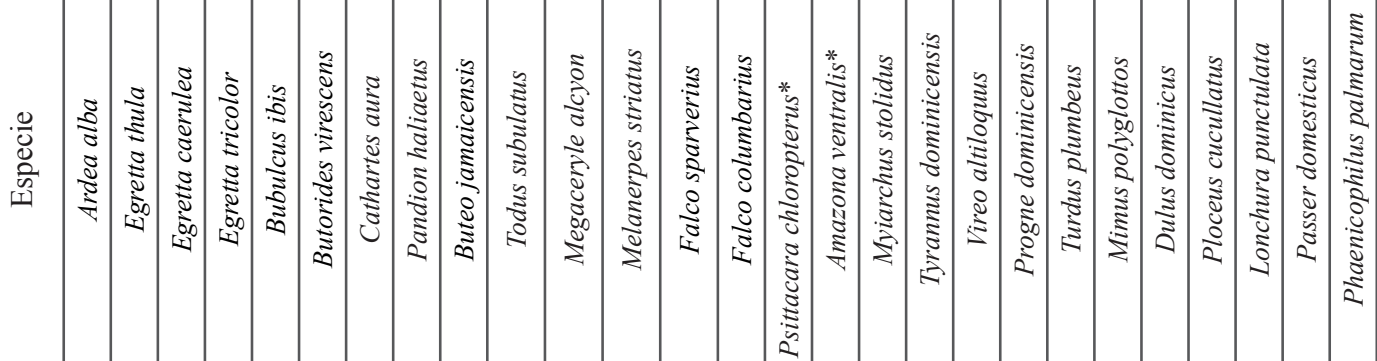

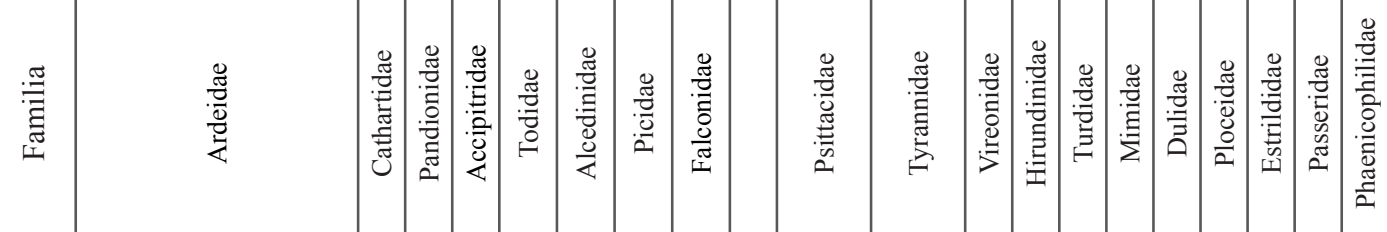




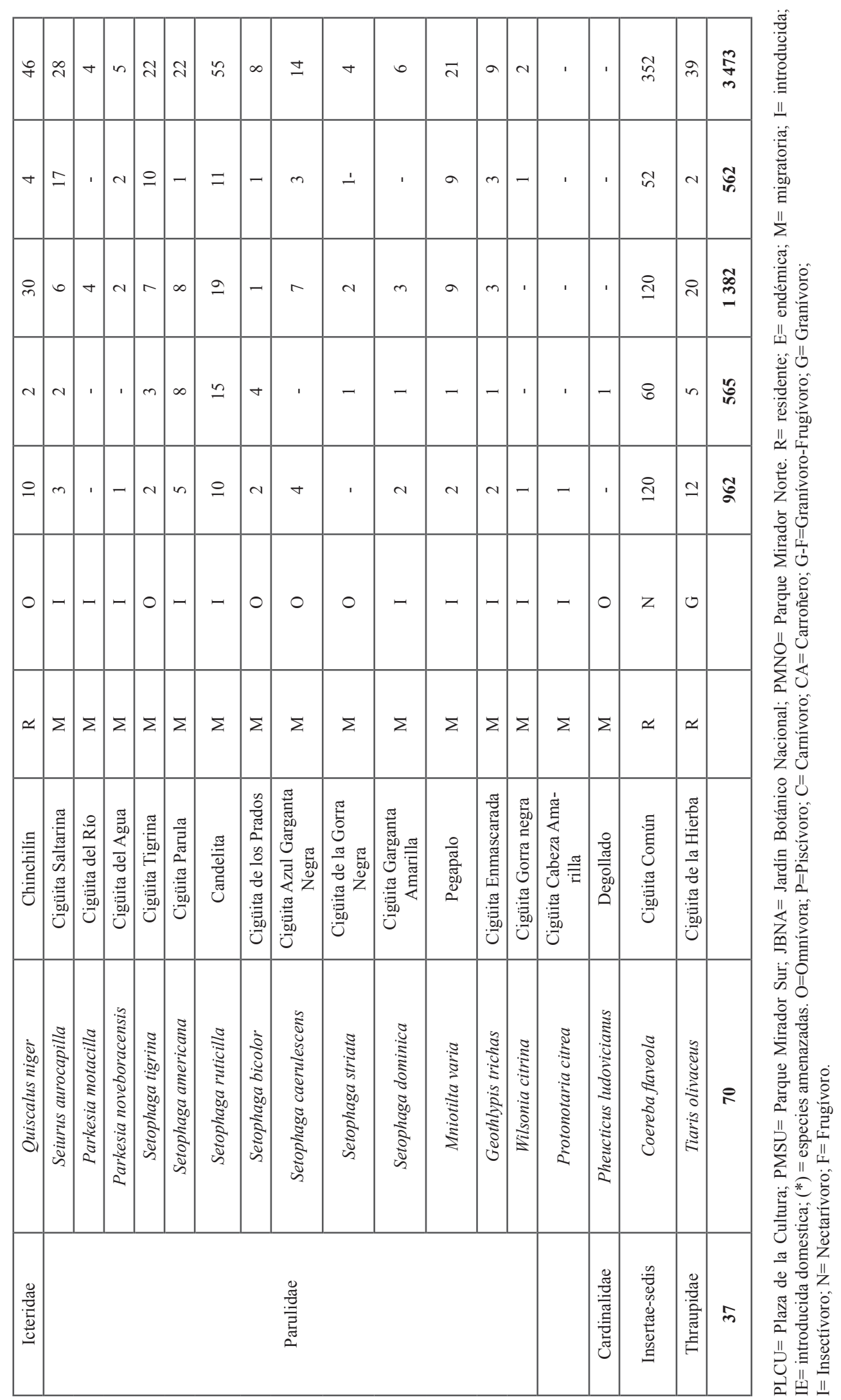


Talla II. Resultados del análisis de los datos en las áreas en estudio

\begin{tabular}{|c|c|c|c|c|}
\hline & PLCU & PMSU & JBNA & PMNO \\
\hline Riqueza de especie (S) & 35 & 38 & 47 & 54 \\
\hline Diversidad de Shannon (H') & 2.3 & 2.4 & 3.00 & 3.30 \\
\hline Equidad de Pielou (J') & 0.70 & 0.78 & 0.86 & 0.96 \\
\hline Dominancia de Simpson ( $\lambda$ ) & 0.07 & 0.06 & 0.08 & 0.05 \\
\hline Reciproco de Simpson (D-1) & 0.89 & 0.93 & 0.92 & 0.94 \\
\hline Abundancia relativa & 0.823 & 0.835 & 1 & 0.998 \\
\hline
\end{tabular}

PLCU= Plaza de la Cultura; PMSU= Parque Mirador Sur; JBNA= Jardín Botánico Nacional; PMNO= Parque Mirador Norte.

\section{AGRADECIMIENTOS}

Al Museo Nacional de Historia Natural "Prof. Eugenio de Jesús Marcano” por el auspicio y financiamiento de este trabajo. Agradecimientos especiales a mis compañeros Francisco Paz, Gabriel de los Santos, Katihuska Rodríguez, Solanlly Carrero, Miguel Nuñez, Patricia Torres y Cristian Marte, por toda la colaboración en el trabajo de campo. A Carlos Suriel por su apoyo y sugerencias. A los revisores anónimos del artículo, quienes hicieron importantes observaciones que permitieron enriquecerlo.

\section{LITERATURA CITADA}

Banks, R., K. Barker, C. Cicero, J. Dunn, A. Kratter, P. Rasmussen, J. Remsen, J. Rising y D. Stotz. 2005. Forty-Sixth Supplement the American Ornithologists' Union Check-List of North American Birds. The American Ornithologists' Union. The Auk, 122 (3): 1026-1031.

Banks, R., C. Cicero, J. Dunn, A. Kratter, P. Rasmussen, J. Remsen, J. Rising y D. Stotz. 2006. Forty-Seventh Supplement to the American Ornithologists' Union Check-List of North American Birds. The Auk, 123 (3): 926-936.

Banks, R., K. Barker, C. Cicero, J. Dunn, A. Kratter, P. Rasmussen, J. Remsen, J. Rising y D. Stotz. 2007. Forty-Eighth Supplement to the American Ornithologists' Union CheckList of North American Birds. The Auk, 124 (3): 1109-1115.

Banks, R., T. Chesser, C. Cicero, J. Dunn, A. Kratter, I. Lovette, P. Rasmussen, J. Remsen, J. Rising, D. Stotz, y K. Winker. 2008. Forty-Ninth Supplement to the American Ornithologists' Union Check-list of North American Birds. The American Ornithologists' Union. The Auk, 125 (3): 758-768.

Carbón-Ramírez, P., I. Zuria y M. Romero-González. 2011. Riqueza, abundancia y dinámica espacio temporal de la comunidad de aves de la ciudad universitaria, Universidad Autónoma del Estado de Hidalgo, Pachuca, México. Canto del Centzontle, 2 (1): 29-47. 
Castillo Palacios, L., L. Castañeda Córdoba y Z. Quintero-Carlos. 2014. Aves del Campus de la Universidad Nacional Agraria la Molina (Lima, Perú)-Una Revisión de su distribución y diversidad desde 1992 al 2010. Ecología aplicada, 13 (2).

Cárdenas, G., C. Harvey, M. Ibrahim y B. Finegen. 2003. Diversidad y riqueza de aves en diferentes hábitats en un paisaje fragmentado en Cañas Costa Rica. Agroforestería en las Américas, vol. $10 \mathrm{~N}^{\mathrm{O}} 39-40$.

Chance, J. F. y J. J. Walsh. 2006. Urban effects on native avifauna: ariew. Landscape and Urban Planning, 74: 46-69.

Chesser, T., R. Banks, C. Cicero, J. Dunn, A. Kratter, P. Rasmussen, I. Lovette, J. Remsen, J. Rising, D. Stotz, y K. Winker. 2009. Fiftieth Supplement to the American Ornithologists' Union Check-list of North American Birds. The American Ornithologists' Union. The Auk, 126 (3): 705-714.

Chesser, T., R. Banks, K. Barker, C. Cicero, J. Dunn, A. Kratter, I. Lovette, P. Rasmussen, J. Remsen, J. Rising, D. Stotz, y K. Winker. 2010. Fifty-First Supplement to the American Ornithologists' Union Check-List of North American Birds. The American Ornithologists' Union. The Auk, 127 (3): 726-744.

Chesser, T., R. Banks, C. Cicero, J. Dunn, A. Kratter, I. Lovette, A. Navarro, P. Rasmussen, J. Remsen, J. Rising, D. Stotz y K. Winker. 2014. Fifty-Fifth Supplement to the American Ornithologists 'Union Check-List of North American Birds. The Auk, 131: CSi-CSxv.

Chesser, T., K. Burns, C. Cicero, J. Dunn, A. Kratter, I. Lovette, A. Navarro, P. Rasmussen, J. Remsen, J. Rising, D. Stotz y K. Winker. 2017. Fifty-eighth supplement to the American Ornithologists 'Union Check-List of North American Birds 'Union Check-List of North American Birds. The Auk, 134: pp 751-773.

Fernández-Juricic, E y J. Jokimäki. 2001. A habitat island approach to conserving birds in urban landscape: case studies from southern and northern Europe. Biodiversity and conservation, 10: 2023-2043.

González-Salazar, C., E. Martínez-Meyer y G. López-Santiago. 2014. A hierarchical classification of trophic guilds for North American birds and Mammals. Revista Mexicana de Biodiversidad, 85: 931-941.

Hinsley, S. A., P. E. Bellamy, I. Newton y T. H. Sparks. 1995. Habitat and landscape factors influencing the presence of individual bird species in woodland fragments. Journal of Avian Biology, 26: 94-104.

Lancaster, R. K y W. E. Rees. 1979. Bird communities and structure of urban habitats. Canadian Journal of Zoology, 57: 2358-2368.

Latta, S., C. Rimmer, A. Keith, J. Wiley, H. Raffaele, K. Mcfarland y E. Fernández. 2006. Aves de la República Dominicana y Haití. Princeton University Press. VII+258 pp.

Latta, S. y K. Wallace. 2012. Ruta Barrancolí. National Aviary, 241 pp. 
Lorenzo, R., J. Núñez y T. Lara. 2014. Fauna. http:/www.adn.gob.do/index.php?view= list\&slug=santo-domingoverde\&option=com_docman\&layout=table\&Itemid=641. Accesado: 03/04/2018.

Maragliano, R. E., L. J. Marti, L. M. Ibáñez y D. Montalt. 2009. Comunidades de aves urbanas de Lavallol, Buenos Aires, Argentina. Acta Zoológica Lilloana, 53 (1-2): 108-114.

Mejía, M. y R. García. 1994. El Jardín Botánico Nacional. Editora Corripio, Santo Domingo, $60 \mathrm{pp}$.

Miembros del Consejo Nacional de Asuntos Urbanos. 2007. Informe GEO Santo Domingo, $242 \mathrm{pp}$.

Moreno, C. E. 2001. Método para medir la biodiversidad. M\&T-Manuales y Tesis SEA, vol.1. Zaragoza, 84 pp.

Ralph, C. J., S. Droege y J. R. Sauer. 1995. Managing and Monitoring Birds Using Point Counts: Standards and Applications. Pages 161-168 in C. J. Ralph, J. R. Sauer, and S. Droege, Eds. Monitoring Bird Populations by Point Counts, USDA Forest Service, Pacific Southwest Research Station, General Technical Report PSW-GTR-149.

Ralph, C. J., G. R. Geupel, P. Pyle, T. E. Martín, D. F. DeSante y B. Milá. 1996. Manual de métodos de campo para el monitoreo de aves terrestres. Gen. Tech. Rep. PSW-GTR-159. Albany, CA: Pacific Southwest Research Station, Forest Services, U. S. Department of Agriculture, $46 \mathrm{pp}$.

Vázquez Plass, E. O y J. M. Wunderle Jr. 2013. Avian distribution along a gradient of urbanization in northeastern Puerto Rico. Ecological Bulletins, 54: 141-156. Wiley-Blackwell, Hoboken, NJ.

[Recibido: 25 de enero, 2018. Aceptado para publicación: 23 de abril, 2018] 